(C) SAGE Publications https://doi.org/10.1177/00219983211021886

\title{
Thermal and dielectric properties of Carbon nanotubes/Graphite/Polyester ternary composites
}

R. Belhimria ${ }^{1}$, Z. Samir ${ }^{1}$, S. Boukheir ${ }^{1,2}$, S. Soreto Teixeira ${ }^{3}$, M. E. Achour ${ }^{1}$, A. AnsonCasaos $^{4}$, J. M. Gonzalez-Dominguez ${ }^{4}$, L. C. Costa ${ }^{3}$, M. El Hasnaoui ${ }^{1, *}$.

${ }^{1}$ Laboratory of Material Physics and Subatomic, Faculty of Sciences, Ibn Tofail University, BP 242, 14000 Kenitra, Morocco

${ }^{2}$ Laboratoire LN2E, Faculté des Sciences, Université Cadi Ayyad, B.P. 2390, 40000 Marrakech, Morocco

${ }^{3}$ I3N and Physics Department, University of Aveiro, 3810-193 Aveiro, Portugal

${ }^{4}$ Instituto de Carboquímica ICB-CSIC, Miguel Luesma Castán 4, 50018 Zaragoza, Spain

*Corresponding author; Mohamed El Hasnaoui: med.elhasnaoui@uit.ac.ma

\section{Abstract}

Binary and ternary composites were synthesized using a polyester matrix reinforced by two types of carbon inclusions, namely, carbon nanotubes (CNT) and graphite (Gt) (CNT/Gt/Polyester). Thermal analyses were performed, using thermogravimetry and differential scanning calorimetry, which allowed us to observe significant changes in glass transition temperatures and degradation temperatures of the composites. Dielectric measurements were performed in a frequency range from $100 \mathrm{~Hz}$ to $1 \mathrm{MHz}$ and temperature from -33 to $107{ }^{\circ} \mathrm{C}$. The dielectric permittivity values of the $\mathrm{CNT} / \mathrm{Gt} /$ Polyester ternary composites, compared to the Gt/Polyester binary composites, indicate that the addition of CNT particles to the Gt/Polyester binary system significantly improved the dielectric permittivity, due to the enhanced interfacial polarization of the host matrix, while the frequency dependence of the electrical modulus spectra revealed a Maxwell-Wagner-Sillars dielectric relaxation process that was found to follow the Cole-Davidson approach.

Keywords: Polyester, Carbon nanotube, Graphite, Thermal analyses, Electrical modulus, Cole-Davidson model. 


\section{Journal of Composite Materials - Lancaster: 00219983211021886 (2021). Copyright}

(C) 2021 Authors. DOI: https://doi.org/10.1177/00219983211021886

\section{Introduction}

Polymer composites with nanostructured carbon fillers have recently received significant interest in research and industry owing to their high conductivity, ease of processing, low weight, etc...[1-4]. They are largely employed in a variety of applications, including flexible conductive materials, antistatic materials, and electromagnetic shielding materials [5-7]. Each type of carbon particles, namely, carbon black (CB), graphite (Gt), carbon fiber (CF), and carbon nanotubes (CNT), possess specific characteristics. For instance, Gt is cheap, but generally has a high percolation threshold, which might affect mechanical properties. CNT has an extremely low percolation threshold due to their high inherent conductivity and large aspect ratio, but they have a high cost for a wide application [8]. The combination of different carbon fillers would be a good option to achieve a balance between properties and cost. Indeed, some polymer blends containing two different carbon fillers (e.g., CF and CB) have a remarkable increase in electrical conductivity, which has been reported in other works [9-14]. It is generally assumed that two types of carbon particles are able to form supporting conductors: those networks in which the CF or the fibrous filler operates at long distances, while the $\mathrm{CB}$ or Gt particulate filler serves as an interconnection between the fibers by forming local conductive paths $[9,10]$.

One of the methods that can provide valuable information on the frequency responses of polymer composites is dielectric spectroscopy. Several relaxation types could be noticed in such heterogeneous systems, namely polymer matrix-related dipolar and Maxwell-WagnerSillars (MWS) relaxations [15-17]. The reason behind the relaxation is the presence of freely immobilized intrinsic fillers (catalyst, impurities...) in the polymer phase. Charges can migrate upon the applied electric field as long as the temperature is high enough to ensure certain mobility in the material. These free carriers are then trapped at the interfaces that separate the two media of different conductivity and permittivity, thus allowing interfacial polarization [18].

The dielectric analysis is a process that measures two basic electrical properties of the material: the capacitance and conductance/or resistance versus frequency. These measurements are then used to calculate the real and imaginary parts $\left(\varepsilon^{\prime}, \varepsilon^{\prime \prime}\right)$ of the complex permittivity. In polymers and polymer composite materials, interfacial polarization is mostly 


\section{Journal of Composite Materials - Lancaster: 00219983211021886 (2021). Copyright}

\section{2021 Authors. DOI: https://doi.org/10.1177/00219983211021886}

occurring as additives, fillers or impurities migrating to interfaces or sites where discontinuity of dielectric properties makes these systems heterogeneous. Generally, interfacial relaxation is masked by the phenomenon of conductivity and dielectric permittivity may reach high values at low frequencies $[19,20]$. To address this concern in the study of interfacial polarization, it was agreed to adopt the formalism of the "electrical modulus", originally initiated by McCrum et al. [21]. Macedo et al. [22], who used it for the inquiry of electrical relaxation phenomena in glass ionic conductors, and El Bachiri et al. [23] used it for analyzing the relaxation process in ferroelectric ceramics. It was also used in polymers and biopolymers to study their relaxation conductivity [24-26].

In this paper, ternary composite materials were prepared by mixing carbon nanotubes (CNT) and graphite (Gt) particles into the polyester matrix. We focus our interest to investigate the influence of the CNT/Gt content on the thermal and dielectric properties of the composites. The thermal properties were analyzed by thermogravimetry (TGA) and differential scanning calorimetry (DSC). The dielectric properties were analyzed using the complex permittivity and the electrical modulus, depending on the frequency, temperature, and fillers concentration in the polymer matrix. Furthermore, Nyquist plots were used to interpret the electrical modulus spectra of all the studied samples.

\section{Experimental}

\section{Materials and samples preparation}

The used polymer is an unsaturated polyester resin 154TB provided from Cray Valley/Total, USA, containing $31 \%$ of styrene monomer and requiring $30 \mathrm{~min}$ for gelation at room temperature. The Graphite (Gt) sample consists of natural graphite flake from Madagascar afforded by CRPP Center, Bordeaux University in France. The grain size ranges from 20 to $100 \mu \mathrm{m}$, the density is $2.09-2.26 \mathrm{~g} / \mathrm{cm} 3$, and the electrical resistivity is from 5 to $8 \Omega . \mu \mathrm{m}$ at 20 ${ }^{\circ} \mathrm{C}$. Multi-walled carbon nanotubes (CNT) were supplied by Cheap-Tubes, USA; their average diameter is about $50 \mathrm{~nm}$, the length ranges from 10 to $20 \mu \mathrm{m}$ and purity is above 95\%. The CNT/Gt/Polyester composite samples were prepared with an $8 \mathrm{wt} \%$ total content in the fillers $(\mathrm{CNT}+\mathrm{Gt})$, which were mixed at different ratios. The CNT/Gt mixtures were mixed with the polyester in different concentrations and stirred at room temperature. The 


\section{Journal of Composite Materials - Lancaster: 00219983211021886 (2021). Copyright}

\section{(C) 2021 Authors. DOI: https://doi.org/10.1177/00219983211021886}

$\mathrm{CNT} / \mathrm{Gt}$ mixtures were first dispersed in the liquid phase methanol under magnetic stirring to reduce the aggregates size. After complete evaporation of methanol at room temperature, the obtained CNT/Gt powder was then directly added to the polyester. Finally, the mixture was injected into sample molds. The samples were removed from the mold when they achieved gelation, after a few hours at room temperature, and then they were left to rest in order to reach the complete polymerization. The prepared samples of ternary composites with detailed concentration values are tabulated in Table 1.

\section{Thermal characterization}

Thermogravimetric analysis (TGA) was performed in a Netzsch TG209 F1 Libra balance. Experiments were carried out under nitrogen and synthetic air at $20{ }^{\circ} \mathrm{C} / \mathrm{min}$. Differential scanning calorimetry (DSC) measurements were performed using a Mettler DSC-823e equipment, under a constant flow of $\mathrm{N}_{2}(60 \mathrm{~mL} / \mathrm{min})$, on $\sim 10 \mathrm{mg}$ sample aliquots, and placed into standard $40 \mu \mathrm{L}$ aluminum crucibles. The device was calibrated using an indium standard. The heating program was preceded by erasing thermal history. For this aim, samples were subjected to dynamic heating from room temperature to $200{ }^{\circ} \mathrm{C}$ at $10^{\circ} \mathrm{C} / \mathrm{min}$ heating rate, held at $200{ }^{\circ} \mathrm{C}$ for $5 \mathrm{~min}$, and cooled down to room temperature at $-10{ }^{\circ} \mathrm{C} / \mathrm{min}$. Subsequently, the heating cyclic program applied to the samples was from -50 to $200{ }^{\circ} \mathrm{C}$, with a heating/cooling rate of $10{ }^{\circ} \mathrm{C} / \mathrm{min}$. Glass transition temperature $\left(\mathrm{T}_{\mathrm{g}}\right)$ values were taken as the inflection point during the cooling step of the last cycle. Average Tg values and sample standard deviations were calculated from 4 measurements for each material.

\section{Dielectric characterization}

Dielectric measurements were performed using an Agilent 4294A Impedance Analyzer in the frequency range from $100 \mathrm{~Hz}$ to $1 \mathrm{MHz}$. Isothermal measurements were carried out in the range from -33 to $107^{\circ} \mathrm{C}$. The sample surfaces were polished and covered with a thin layer of silver paint. In the electrical model, each sample is realistically represented by a parallel combination of a capacitor $C_{p}$ and a resistance $R_{p}$, which permits the calculation of the real, $\varepsilon^{\prime}(F)$, and imaginary, $\varepsilon^{\prime \prime}(F)$, parts of complex permittivity, $\varepsilon^{*}(F)=\varepsilon^{\prime}(F)-j \varepsilon^{\prime \prime}(F)$, using the following relations: 
CC 2021 Authors. DOI: https://doi.org/10.1177/00219983211021886

$$
\begin{gathered}
\varepsilon^{\prime}(F)=\frac{d}{A} \frac{1}{\varepsilon_{o}} C_{p}(F) \\
\varepsilon^{\prime \prime}(F)=\frac{d}{A} \frac{1}{\varepsilon_{o}} \frac{1}{2 \pi F R_{p}(F)}
\end{gathered}
$$

where $F$ is the frequency and $\varepsilon_{0}$ is the permittivity of free space, $d$ and $A$ are the sample thickness and electrode surface area, respectively. The use of electrical modulus offers some advantages in interpreting bulk relaxation processes, at low frequencies. The electrical modulus, $\mathbf{M}^{*}(\mathrm{~F})$, is defined in terms of the reciprocal complex permittivity as $M^{*}(F)=1 / \varepsilon^{*}(F)=M^{\prime}(F)+j M^{\prime \prime}(F)$. The real, $\mathbf{M}^{\prime}$, and imaginary, $\mathbf{M}^{\prime \prime}$, parts of the electrical modulus are calculated from $\varepsilon^{\prime}(\mathrm{F})$ and $\varepsilon^{\prime \prime}(\mathrm{F})$ via equation (3). The estimated relative error of the electrical measurements is less than $5 \%$.

$$
M^{*}(F)=\frac{\varepsilon^{\prime}(F)}{\mathcal{E}^{\prime}(F)^{2}+\varepsilon^{\prime \prime}(F)^{2}}+j \frac{\varepsilon^{\prime \prime}(F)}{\mathcal{E}^{\prime}(F)^{2}+\varepsilon^{\prime \prime}(F)^{2}}
$$

\section{Results and discussion}

\section{Thermal analysis}

DSC experiments of the neat polyester (S1) and its composites S2, S3, and S4 are shown in Figure 1. As can be seen, all samples exhibited a clear glass transition $\left(\mathrm{T}_{\mathrm{g}}\right)$, particularly during the cooling down. The $\mathrm{T}_{\mathrm{g}}$ values and their standard deviations were calculated and listed in the inset of Figure 1. Certain changes can be observed in the $T_{g}$ depending on both filler concentrations. The $\mathrm{T}_{\mathrm{g}}$ value of the composite $\mathrm{S} 2$ is $11{ }^{\circ} \mathrm{C}$ higher than that of the pure polyester. This could be related to an increase in the rigidity of the system, as a consequence of the graphite presence, which would hinder the mobility of polymer chains [27]. Such an imposed motion restriction is indicative of a reinforcing effect. Then, the substitution of a $0.5 \%$ graphite for CNTs (sample S4) seems to palliate this effect, suggesting that CNTs would not impose such a restriction in the motion of polymer chains as compared to graphite

TGA experiments were conducted to study the thermal and thermo-oxidative stability of the neat polyester (S1) and its composites S2, S3, and S4 (Figure 2). In both nitrogen and synthetic air atmospheres cases, weight losses start above $100{ }^{\circ} \mathrm{C}$, indicating that all the materials bear low levels of moisture. Moderate weight losses occur in both inert and 


\section{Journal of Composite Materials - Lancaster: 00219983211021886 (2021). Copyright}

\section{(C) 2021 Authors. DOI: https://doi.org/10.1177/00219983211021886}

oxidative atmospheres in the range of $150-300{ }^{\circ} \mathrm{C}$. An abrupt loss is observed around $350-420$ ${ }^{\circ} \mathrm{C}$ due to degradation of the polymer. Polyester and polystyrene chains break to form a primary char, releasing $\mathrm{CO}, \mathrm{CO} 2$, styrene and phthalic anhydride as the main gas products [28]. In addition, the degradation under oxidative atmospheres revealed another weight loss at a high temperature around $510-550{ }^{\circ} \mathrm{C}$, which is associated with the char oxidation [28].

In order to look for a deeper analysis and identify reference temperature in TGA curves, we calculated the derivative plots (DTG). As an example, Figure 3 compares the plot of weight loss and its derivatives for the neat polyester (S1) under nitrogen (Figure 3a) and synthetic air atmospheres (Figure 3b). The as determined values of the characteristic degradation temperatures of pure polyester (S1) and its composites (S2, S3 and S4) are summarized in Table 2. The inspection of these results indicates that i) the thermal degradation takes place as two overlapping processes under nitrogen, providing the two temperatures $T_{d 1}$ and $T_{d 2}$, as it was previously observed in conducting polymer composites [29]. In contrast, the degradation under oxygen takes place with at least an extra step, i.e, in addition to two successive degradations at $\mathrm{T}_{\mathrm{d} 1}$ and $\mathrm{T}_{\mathrm{d} 2}$, a third degradation is revealed at $\mathrm{T}_{\mathrm{d} 3}$. ii) As a general trend, the composites show almost identical $\mathrm{T}_{\mathrm{d}}$ values, while most of them are higher than that of the neat polyester. Under the air atmosphere, the maximum stability is observed for the $8 \% \mathrm{Gt}$ (S2) composite without CNT.

It can be concluded that the thermal stability of the resin substantially improves with the addition of the fillers, particularly under the oxidative atmosphere. This effect might be associated with the thermal dissipation and radical trapping abilities of the carbon fillers [3032]. In fact, the thermal transport through the fillers hinders local heating, while radical chain reactions are soon terminated by reaction with carbon nanostructures. Another contribution to the thermal stability might come from an improvement in the polymer barrier properties (a decrease in the gas permeability) [33]. If the presence of the filler delays the evolution of gas products, the degradation temperature should increase. It has been found that the change in barrier properties using graphene fillers is larger than with nanotubes [33]. From the thermal analyses performed in this study, we noticed that graphite fillers would be a good asset for improving thermal and thermo-oxidative resistance of polyester resins, as well as increasing 


\section{Journal of Composite Materials - Lancaster: 00219983211021886 (2021). Copyright}

(C) 2021 Authors. DOI: $\underline{\text { https://doi.org/10.1177/00219983211021886 }}$

the rigidity of the system, while CNT particles show a compensating effect of the chain motion restriction induced by graphite, as for the decrease in $\mathrm{Tg}$.

\section{Dielectric permittivity analysis}

Comparative plots of the frequency dependence of the real $\left(\varepsilon^{\prime}\right)$ and imaginary $\left(\varepsilon^{\prime \prime}\right)$ parts of the complex permittivity of the neat polyester matrix (S1) and its composites ( $22, \mathrm{~S} 3$, and $\mathrm{S} 4)$ are shown in Figure 4 ( $\mathrm{a}$ and $\mathrm{b}$ ), at room temperature. We can observe that both $\varepsilon^{\prime}$ and $\varepsilon^{\prime \prime}$ increased with the incorporation of $8 \%$ of Gt particles and this increases became more significant with the substitution of Gt by CNT particles. The high improvements of dielectric permittivity at low frequency can be attributed to the interfacial polarization, known as Maxwell-Wagner-Sillars (MWS) effect [34, 16]. This effect appeared in heterogeneous media due to the accumulation of charges at the interfaces. Generally, the dielectric permittivity of polymer composites can be improved by a further increase in the Gt loading. But, in our case, the mechanical properties of composites greatly decline due to the high loading of Gt fillers and regulating the dielectric permittivity by adjusting the filler content become very difficult.

Thanks to the CNT unique properties with extremely small size (compared to Gt), high conductivity and high mechanical strength, the incorporation of a small content of CNT strongly improves dielectric permittivity. Therefore, in order to further enhance the dielectric permittivity by using almost the same filler content, in this study, the CNT/Gt/Polyester three phase composites were prepared. We note that the substitution of Gt with CNT in the CNT/Gt/polyester composites (Figure 4 (a) and (b)) increased relatively the dielectric constants at low frequencies, and this is because of the largest difference of the electrical conductivity between the filler and the polymer resulting in a stronger interfacial polarization compared with the Gt/Polyester composite. The significant improvement in the dielectric permittivity that can be achieved with a relatively low loading of the CNT is due to the high surface area, and high aspect ratio, leading to the improved interfacial polarizations of the Gt/Polyester. This happens at low frequency applied alternating voltage [35], as the mobile charges may easily hop out of the low energy sites, which leads to the increase of the polarization of composites and therefore causes the increase of both real and imaginary parts of the complex permittivity $[36,37]$. As the frequency increases, the oscillations of mobile 


\section{Journal of Composite Materials - Lancaster: 00219983211021886 (2021). Copyright}

\section{(C) 2021 Authors. DOI: https://doi.org/10.1177/00219983211021886}

charges with the macromolecular structure of composite increase, resulting in the reduction of the dielectric permittivity.

In order to analyze the temperature effect on dielectric permittivity of the neat polyester and its composites, we have represented in Figure 5, the imaginary part, $\varepsilon "$, versus temperature of sample S3 at different frequencies (Figure 5a) and comparative curves of all samples at $5 \mathrm{kHz}$ (Figure $5 b$ ). It can be observed that, $\varepsilon "$ presents remarkable variations at the glass transition temperature $(\mathrm{Tg})$; below this temperature $\varepsilon "$ is slightly constant for samples S1 and S2 and shows small decreases for samples $\mathrm{S} 3$ and $\mathrm{S} 4$, this can be explained by the fact that the CNTs existing in samples S2 and S3 are aligned with the effect of an applied field reduces the phase lag [38], thus decreasing the dielectric loss of the composite, and above the $\mathrm{Tg}, \varepsilon$ " showed a significant increase at several frequencies, which can be ascribed to the orientational polarization connected to the thermal motion of molecules. As the temperature is increased, the orientation of the dipoles is facilitated and the imaginary part of the dielectric permittivity increases as well [39]. On the other hand, it can also be seen that the dielectric constant decreases with frequency, as the dipoles are unable to orientate themselves with the applied field. While $\varepsilon^{\prime \prime}$ increases with the increasing of fillers, and this is related to the increase of mobility and polarization of space charge carriers.

\section{Electrical modulus analysis}

The electrical modulus formalism has been used in several works for interpreting the relaxation processes appearing in the dielectric responses of composites materials based on polymeric matrix reinforced with conducting fillers [40,41]. Figure 6 shows the imaginary part, M", of the complex modulus as a function of frequency for neat polyester (S1) and its composites (S2, S3, and S4), in the range of temperatures below and above the glass transition point $\mathrm{T}_{\mathrm{g}}$. Analysis of these figures yields the following results: i) for the neat polyester matrix (S1) no relaxation can be detected, ii) the incorporation of $0.8 \% \mathrm{Gt}$ particles (S2) to this matrix permits to identify of a remarkable relaxation and this relaxation appears more clearly with the addition of $0.2 \% \mathrm{CNT}$ particles to the Gt/polyester binary composite (S3), meaning that the conducting particles are the responsible for dielectric relaxations, and iii) these relaxations depend strongly on temperature and shift toward high frequencies as the CNT particle concentration increases. As a result, and compared to the investigation of Psarras et 


\section{Journal of Composite Materials - Lancaster: 00219983211021886 (2021). Copyright}

\section{(C) 2021 Authors. DOI: https://doi.org/10.1177/00219983211021886}

al. [42] who presented a study on iron particles loaded-polymer composites, we attributed this relaxation to the interfacial polarization or so-called Maxwell-Wagner-Sillars (MWS) effect that results from the charge accumulations inside the polyester matrix confirming the behavior of the permittivity spectra discussed above mentioned $[34,16]$. We note that for the composites S2, S3 and S4, the value of $\mathrm{M}^{\prime}$ drop to zero at low frequencies, indicating that the electrode polarization has a negligible effect in our case $[43,44]$.

\section{Cole-Davidson modeling}

As observed in Figure 6, the asymmetrical spectra of M"(F) indicates that the Debye model is inappropriate to describe the relaxation occurring in our composites, and should be replaced by a frequency-dependent electrical modulus, written as [45]:

$$
M^{*}(\omega)=M_{\infty}^{\prime}\left[1-\int_{0}^{\infty} e^{j \omega t}(-d \phi(t) / d t) d t\right]
$$

where the function $\phi(t)$ is the depolarization function. The real $\left(\mathrm{M}^{\prime}\right)$ and imaginary $\left(\mathrm{M}^{\prime \prime}\right)$ parts of the complex electrical modulus, according to the Cole-Davidson model bear the following expressions [46]:

$$
\begin{aligned}
M^{\prime}(\omega) & =\frac{M_{\infty} M_{s}\left[M_{s}+\Delta M(\cos \phi)^{\beta} \cos (\beta \phi)\right]}{M_{s}^{2}+\Delta M(\cos \phi)^{\beta}\left[2 M_{s} \cos (\beta \phi)+\Delta M(\cos \phi)^{\beta}\right]} \\
M^{\prime \prime}(\omega) & =\frac{M_{\infty} M_{s} \Delta M(\cos \phi)^{\beta} \sin (\beta \phi)}{M_{s}^{2}+\Delta M(\cos \phi)^{\beta}\left[2 M_{s} \cos (\beta \phi)+\Delta M(\cos \phi)^{\beta}\right]}
\end{aligned}
$$

where $M_{s}$ and $M_{\infty}$ are the real part of electric modulus at low- and high-frequency sides of the relaxation, respectively, and $\Delta \mathrm{M}=M_{\infty}-M_{s} . \tau$ is the relaxation time, $\phi$ is an angle defined as $\operatorname{tg} \phi=\omega \tau . \beta$ is the fractional shape parameter between 0 and 1 , describing the skewing of the dielectric function, reflecting the dipole interaction. We performed a fit of the experimental data using the above equations (5) and (6) from which we extracted the parameters $\beta, \tau$, and $\Delta M$. Figure 7 shows an example of $\mathrm{M}^{\prime \prime} \mathrm{vs} . \mathrm{M}^{\prime}$ for the data of sample $\mathrm{S} 3$, at different temperatures, together with their simulations fits. Table 3 summarizes the obtained values of relaxations parameters for the binary S2 (Gt/polyester) and ternary S3 (CNT/Gt/polyester) samples. Several remarks and conclusions can be extracted from the analysis of these results: 
C) 2021 Authors. DOI: https://doi.org/10.1177/00219983211021886

i) the values of $\beta$ are in the range 0.05-0.09 for sample $S 2$ and in the range 0.32-0.44 for sample S3 showing its significant increases as CNT particles added into the Gt/polyester binary composite, meaning that more interactions are produced with the existence of CNT, ii) the increases of modulus strength, $\Delta \mathrm{M}$, from 0.05 to 0.09 with temperature for sample $\mathrm{S} 2$ (Gt/polyester) and its increases from (0.05-0.09) in the case of S2 to (0.11-0.12) in the case of S3 (CNT/Gt/polyester) are explained by a good adhesion of the Gt and CNT particles with the polyester matrix $[47,48]$

\section{Conclusion}

This paper presents studies of thermal and dielectric properties of binary and ternary composites based on the dispersion of Gt and CNT particles in a polyester matrix. Thermal analysis showed that Gt fillers would be a good asset for improving thermal and thermooxidative resistance of polyester resins, and increasing in the glass transition temperature $\left(\mathrm{T}_{\mathrm{g}}\right)$. The addition of small quantities of CNT fillers decreased the thermal degradation temperature and $\mathrm{Tg}$ values with respect to the binary Gt/polyester composite. Dielectric analyses were carried out in the frequency range from $100 \mathrm{~Hz}$ to $1 \mathrm{MHz}$ and temperature below and above $\mathrm{T}_{\mathrm{g}}$. The dielectric permittivity analysis showed that the addition of CNT to the Gt/polyester composite leading to an enhancement of interfacial polarization of composite, while the electric modulus exhibits a typical dielectric relaxation process that attributed to the MaxwellWagner-Sillars relaxation, and the behavior of this relaxation is analyzed using the ColeDavidson model.

\section{Acknowledgements}

The authors thank FEDER funds through the COMPETE 2020 Program and National Funds through FCT - Portuguese Foundation for Science and Technology under the project UID/CTM/50025/2019 and acknowledge the financial support from the Diputación General de Aragón under project T03_20R (Grupo Reconocido).

\section{References}

1. Zhang W, Dehghani-Sanij AA and Blackburn RS. Carbon based conductive polymer composites J Mater Sci 2007; 42: 3408-3418. 


\section{Journal of Composite Materials - Lancaster: 00219983211021886 (2021). Copyright}

CC 2021 Authors. DOI: https://doi.org/10.1177/00219983211021886

2. Gangopadhyay R, De A. Conducting polymer nanocomposites: A brief overview. Chem Mater 2000; 12: 608-622.

3. Jagur-Grodzinski J. Electronically conductive polymers. Polym Adv Technol 2002 ; 13: 615-625.

4. Kalaitzidou K, Fukushima H and Drzal LT. A new compounding method for exfoliated graphite-polyropylene nanocomposites with enhanced flexural properties and lower percolation threshold. Compos Sci Technol 2007; 67: 2045-2051.

5. Gordeyev SA, Ferreira JA, Bernardo C A, et al. A promising conductive material: highly oriented polyropylene filled with short vapour-grown carbon fibres. Mater Lett 2001; 51: 32-36.

6. Baughman RH, Zakhidov AA and De Heer WA. Carbon nanotubes-the route toward applications. Science 2002; 297: 787-792.

7. Chung DDL. Electromagnetic interference shielding effectiveness of carbon materials Carbon 2001; 38: 279-285

8. Abazine K, Anakiou H, El Hasnaoui M, et al. Electrical conductivity of multiwalled carbon nanotubes/polyester polymer nanocomposites. J Compos Mater 2016; 50: 32833290 .

9. Clingerman LM, Weber EH, King JA, et al. Synergistic effect of carbon fillers in electrically conductive nylon 6, 6 and polycarbonate based resins. Polym Compos 2002; 23: 911-924.

10. Thongruang W, Spontak RJ and Balik CM. Correlated electrical conductivity and mechanical property analysis of hight-density polyethylene filled with graphite and carbon fiber. Polymer 2002; 43: 2279- 2286.

11. Drubetski M, Siegmann A, Nakis M. Hybrid particulate and fibrous injection molded composites: Carbon black/carbon fiber/polypropylene systems. Polym Compos 2005; 26: 454-464.

12. Lee JH, Kim SK and Kim NH. Effects of the addition of multi-walled carbon nanotubes on the positive temperature coefficient characteristics of carbon-black-filled high-density polyethylene nanocomposites. Scr Mater 2006; 55: 1119-1122.

13. Drubetski M, Siegmann A and Nakis M. Electrical properties of hybrid carbon black/carbon fiber polypropylene composites. J Mater Sci 2007; 42: 1-8.

14. EI-Bounia NE and Piccione PM. Rheological and electrical properties of PVDF composites based on carbon black and carbon nanotubes synergy. J Polym Eng 2008; 28: 141-154.

15. Maxwell JC. Electricity and Magnetism, Clarendon press, Oxford, 1892.

16. Wagner KW. Erklärung der dielektrischen Nachwirkungsvorgänge auf Grund Maxwellscher Vorstellungen. Arch. Elektrotech 1914; 2: 371-387.

17. Sillars RW. The properties of a dielectric containing semiconducting particles of various shapes. J Inst Elec Engrs 1937; 80: 378-394. 
(C) 2021 Authors. DOI: $\underline{\text { https://doi.org/10.1177/00219983211021886 }}$

18. El Hasnaoui M, Triki A, Achour ME, et al. Modeling of dielectric relaxation processes of epoxy-resin filled with carbon black particles. Physica B Consens Matter 2014; 433: 6266.

19. Hedvig P. Dielectric Spectroscopy of Polymers. Hilger, Bristol, 1977.

20. Hammami H, Arous M, Lagache $\mathrm{M}$, et al. Experimental study of relaxations in unidirectional piezoelectric composites. Comp Part A Appl Sci Manuf. 2006; 37: 1-8

21. McCrum NG, Read BE and Williams G. Anelastic and dielectric effects in polymeric solid. London 1967; 108-111.

22. El Bachiri A, El Hasnaoui M, Louardi A, et al. Structural and dielectric studies for the conduction mechanism analyses of lithium-niobate oxide ferroelectric ceramics. Physica B Condens Matter 2019; 571: 181-187.

23. Kreit L, Bouknaitir I, Zyane A, et al. Electrical conductivity and dielectric relaxation studies of biocomposites based on green microcrystalline cellulose-reinforced vinyl resin matrix. J Compos Mater 2019; 50: 2801-2808.

24. Srarkweather HW and Avakian P. Conductivity and the electric modulus in polymers. Polym Sci B Polym Phys 1992; 30: 637-641.

25. Chen SA, Liao CS. Conductivity relaxation and chain motions in conjugated conducting polymers: neutral poly (3-alkylthiophenes). Macromolecules 1993; 26: 2810-2816.

26. Boukheir S. Structural, Electrical and Dielectric Properties of Carbon Allotropes - Based Multifunctional Nanocomposites. PhD Thesis, University Cadi Ayyad, Morocco, 2018.

27. Grady BP. Effects of carbon nanotubes on polymer physics. J Polym Sci Part B Polym Phys 2012; 50: 591-623.

28. Kandare E, Kandola BK, Price D, et al. Study of the thermal decomposition of flameretarded unsaturated polyester resins by thermogravimetric analysis and Py-GC/MS. Polym. Degrad. Stabil. 2008; 93: 1996-2006.

29. Aribou N, Barnoss S, El Hasnaoui M, et al. Structural, electrical and thermal properties of composites based on conducting polymer. Jordan J Phy 2020; 13: 157-163.

30. Tarani E, Terzopoulou Z, Bikiaris DN, et al. Thermal conductivity and degradation behavior of HDPE/graphene nanocomposites. J Therm Anal Calorim 2017; 129: 17151726.

31. Melk L and Emami N. Mechanical and thermal performances of UHMWPE blended vitamin E reinforced carbon nanoparticle composites. Compos Part B-Eng 2018; 146: 20-27.

32. Martinez-Morlanes MJ, Castell P, Alonso PJ, et al. Multi-walled carbon nanotubes acting as free radical scavengers in gamma-irradiated ultrahigh molecular weight polyethylene composites. Carbon 2012; 50: 2442-2452.

33. Wen S, Zhang R, Xu Z, et al. Effect of the topology of carbon-based nanofillers on the filler networks and gas barrier properties of rubber composites. Materials 2020; 13: 5416.

34. Abd-El Ghany S, Abd-El Salam MH and Nasr GM. Study of dielectric properties of particulate blends. J Appl Polym Sci 2000; 77: 1816-1821. 
(C) 2021 Authors. DOI: $\underline{\text { https://doi.org/10.1177/00219983211021886 }}$

35. Ali AA and Shaaban MM, Electrical properties and scaling behavior of $\mathrm{Sm}^{3+}$ doped $\mathrm{CaF}_{2}$ bismuth borate glasses. Bull Mater Sci 2011; 34: 491-498.

36. Majhi K, Varma KBR, Dielectric relaxation in $\mathrm{CaO}-\mathrm{Bi}_{2} \mathrm{O}_{3}-\mathrm{B}_{2} \mathrm{O}_{3}$ glasses. Int J Appl Ceram Technol 2010; 7: E89-E97.

37. Naceur $\mathrm{H}$, Megriche $\mathrm{A}$ and $\mathrm{El}$ Maaoui $\mathrm{M}$. Frequency-dependent dielectric characteristics and conductivity behavior of $\mathrm{Sr}_{1-\mathrm{x}}\left(\mathrm{Na}_{0.5} \mathrm{Bi}_{0.5}\right) \mathrm{Nb}_{2} \mathrm{O}_{9}(\mathrm{x}=0.0,0.2,0.5,0.8$, and 1.0) Ceramics Orient J Chem 2013; 29: 937-944.

38. Belcher Cami B. Dielectric Characterization of Mylar and the Effects of Doping Processes. PhD Thesis. M.S., Chemical Engineering, University of New Mexico, 2016.

39. Deger D, Ulutas K. Conduction and dielectric polarization in Se thin films. Vacuum 2004; 72: 307-312.

40. El Hasnaoui M, Triki A, Graca M P F, et al. Electrical conductivity studies on carbon black loaded ethylene butylacrylate polymer composites. J Non Cryst Solids 2012; 358: 2810-2815.

41. Belattar J, Graça MPF, Costa LC, et al. Electric modulus-based analysis of the dielectric relaxation in carbon black loaded polymer composites. J Appl Phys 2010; 107: 124111(16).

42. Psarras GC, Manolakaki E, Tsangaris GM. Dielectric dispersion and ac conductivity inIron particles loaded-polymer composites. Composites: Part A; 2003; 34: 1187-1198

43. Rao KS, Prasad DM, Murali Krishna P, et al. Electrical and electromechanical studies on tungsten-bronze electroceramic: lead potassium dysprosium niobate. Optoelectron Adv Mater Rapid Commun 2007; 1: 510-519

44. Nanda M, Tripathy DK. Physico-mechanical and electrical properties of conductive carbon black reinforced chlorosulfonated polyethylene vulcanizates. Exp Polym Lett 2008; 2: 855-865.

45. Zallen R. The physics of amorphous solids. Wiley, New York, 1985.

46. Tsangaris GM, Psarras GC, Kouloumbi N. Electric modulus and interfacial polarization in composite polymeric systems. J Mater Sci 1998; 33:2027-2037.

47. Ghallabi Z, Rekik H, Boufi S, et al. Effect of the interface treatment on the dielectric behavior of composite materials of unsaturated polyester reinforced by alfa fiber. $J$ NonCryst Solids 2010; 356: 684-687.

48. Arous M, Ben Amor I, Boufi S, et al. Experimental study on dielectric relaxation in alfa fiber reinforced epoxy composites. J Appl Polym Sci 2007; 106: 3631-3640.

\section{Tables captions:}

Table 1: Prepared samples of CNT/Gt/ Polyester ternary composites.

Table 2: Characteristic degradation temperatures of S1, S2, S3 and S4 composites obtained from TGA measurements under nitrogen and synthetic air atmospheres. 
Journal of Composite Materials - Lancaster: 00219983211021886 (2021). Copyright

CC 2021 Authors. DOI: https://doi.org/10.1177/00219983211021886

Table 3: Parameters evaluated by fitting the experimental data according to the ColeDavidson model (Eqs 5 and 6) for the Gt/polyester composite (sample S2) and the CNT/Gt/polyester composite (sample S3).

\section{Figures captions:}

Figure 1: DSC curves of the neat polyester matrix (S1) and its composites (S2, S3 and S4). The inserted table shows the $\mathrm{Tg}$ values with their associated standard deviations.

Figure 2: TGA experiments under (a) nitrogen and (b) synthetic air atmospheres of S1, S2, S3 and S4 specimens.

Figure 3: Mass loss (TG\%) and derivative mass loss (DTG) under nitrogen (a) and synthetic air atmospheres (b) of S1 vs. temperature of the neat polyester.

Figure 4: Frequency dependence of dielectric permittivity (a) real part, (b) imaginary part of $\mathrm{S} 1, \mathrm{~S} 2, \mathrm{~S} 3$ and $\mathrm{S} 4$ composites at room temperature.

Figure 5: Temperature effect on dielectric permittivity $\varepsilon^{\prime \prime}:$ (a) at different frequencies of sample S3, (b) comparison between the neat polyester and its composites, at $5 \mathrm{kHz}$. The marked $\mathrm{T}_{\mathrm{g}}$ represent the values obtained from DSC measurements.

Figure 6: Imaginary part of the electrical modulus, M", as a function of frequency for the composites $\mathrm{S} 1, \mathrm{~S} 2, \mathrm{~S} 3$ and $\mathrm{S} 4$, at different temperatures.

Figure 7: Nyquist representation of the complex electric modulus of the sample S3. Solid lines are produced by fitting experimental points to the Cole-Davidson model (Eqs. 5 and 6). 